\title{
A novel classification to guide total hip arthroplasty for adult acetabular dysplasia
}

\author{
CHEN ZHU ${ }^{1 *}$, MENG-QI CHENG ${ }^{1 *}$, TAO CHENG ${ }^{1}$, RUI-XIANG MA ${ }^{2}$, RONG KONG $^{2}$, \\ YONG-YUAN GUO ${ }^{1}$, HUI QIN ${ }^{1}$, SI FENG SHI ${ }^{1}$ and XIAN-LONG ZHANG ${ }^{1}$ \\ ${ }^{1}$ Department of Orthopaedic Surgery, Shanghai Sixth People's Hospital, Shanghai Jiao Tong University School of Medicine, \\ Shanghai 200233; ${ }^{2}$ Department of Orthopaedic Surgery, Anhui Provincial Hospital, Anhui Medical University, \\ Hefei, Anhui 230001, P.R. China
}

Received January 18, 2013; Accepted April 16, 2013

DOI: $10.3892 /$ etm.2013.1093

\begin{abstract}
In the field of hip arthroplasties, the secondary fixation of the implants depends directly on the quality of the primary stability. A good acetabular fit and metaphyseal filling between the prostheses and implants improve the initial stabilization, and optimize the transmission of forces to the bone. A precise knowledge of the three-dimensional acetabular or femoral shape is essential to the selection of adapted implants. A total of 63 patients diagnosed with developmental dysplasia were analyzed by three-dimensional computed tomography (3DCT), and the preoperative radiographic and 3DCT images were used to assess the acetabular/femoral deformities and variations of the hips. All joints were classified as Crowe type I, and bilateral measurements were taken for 10 patients. The acetabular abnormalities were classified according to the type of deficiency and the section angles of the acetabulum, with 26 hips (36\%) classified as an anterior deficiency, 13 hips (18\%) as a posterior deficiency and 34 hips $(46 \%)$ as a lateral deficiency. The femoral side deformities were divided into three types according to the anteversion angle of the femur. A gradual increase in anteversion angle led to secondary rotational anomalies, and a narrowing of the canal at the isthmus. A total of 35 hips (48\%) were classified as an F1 type deficiency, femur anteversion angle (FAVA) $<30^{\circ} ; 32$ hips (44\%) as F2-type, $30^{\circ} \leq \mathrm{FAVA} \leq 40^{\circ}$, with mild abnormalities of the femoral canal rotation and the diameter of the isthmus; and 6 hips (8\%) as F3 type, FAVA $>40^{\circ}$, with significant abnormalities of the femoral canal rotation and the diameter of the isthmus. This
\end{abstract}

Correspondence to: Professor Xian-Long Zhang, Department of Orthopaedic Surgery, Shanghai Sixth People's Hospital, Shanghai Jiao Tong University, School of Medicine, 600 Yishan Road, Shanghai 200233, P.R. China

E-mail: zxl40@vip.sina.com

*Joint senior authorship

Key words: adult, developmental dysplasia, total hip replacement, three-dimensional, computed tomography novel classification for adult acetabular dysplasia may provide a useful guide for surgery, and enable an improved selection of a suitable prosthesis.

\section{Introduction}

Acetabular dysplasia (AD) is a developmental dysplasia of the hip (DDH), and is also known as hip joint instability. The characteristic pathological change in AD is a shallow acetabulum that leads to insufficient acetabular containment and coverage of the femoral head; however, radiographic observations have demonstrated that the femoral head remains in the true acetabulum (1). Studies from China have revealed that 50-60\% of the patients who received a total hip arthroplasty (THA) suffered from osteoarthritis (OA) secondary to hip dysplasia, and a large number of adult AD patients ultimately undergo a total hip replacement $(2,3)$. It has previously been suggested that the femoral and acetabular anatomical malformations that are apparent with AD increase gradually, in correlation with femoral head displacement (4). Since the patients with these anatomical malformations rarely develop further hip subluxations and dislocations, the majority of doctors do not consider the disorder to be a significant disability. However, anatomical variations of the acetabulum and proximal femoral medullary cavity are irregular (5), and preoperative X-rays do not identify all patients with AD; the correlation of the X-ray results with intraoperative findings varies greatly. A femoral neck fracture with AD is easily missed in clinical practice, and often leads to postoperative dislocation (6). The Crowe classification describes the proximal migration of the femoral head, regardless of the acetabular deformity, and assumes that there is a direct interrelation between the extent of the migration and the severity of disease (7). By contrast, the Hartofilakidis classification relies on the anatomy of the acetabulum, as encountered during surgery (8). However, the two classifications are not always valid, since the anatomy of the acetabulum and femur is variable, and the extent of migration is not a definite criterion for judging the type of dysplasia $(8,9)$. Therefore, these classifications have limited uses as surgical guides, and for the selection of a suitable prosthesis. Furthermore, there is no specialized classification for mild DDH, such as AD. 
With the increasing prevalence of THA, the incidence of adverse results, such as a fracture in the region surrounding the prosthesis and dislocation, has increased at follow-up. These adverse effects are often correlated with improper intraoperative management, most notably the implantation of a conventional prosthesis into an abnormal medullary cavity (10). The correct placement of a suitable prosthesis is the sole method of preventing adverse effects, and ensuring the long-term stability of the prosthesis. Thus, a more effective clinical classification is required to guide surgery. Following an analysis of previous studies, we propose, in the present study, a novel method of assessing acetabular and femoral deformities.

\section{Materials and methods}

Patients. From 2007 to 2011, 63 consecutive patients who were diagnosed with OA secondary to developmental dysplasia, or femoral neck fracture with developmental dysplasia, and who would accept a THA, were treated at Shanghai Sixth People's Hospital (Shanghai, China). The patient cohort consisted of 14 males and 49 females, with a mean age of $55.6 \pm 12.5$ years (range, 18-83 years). A total of 55 were diagnosed with OA, and eight with a femoral neck fracture. Patients who had undergone acetabular or femoral osteotomies or who suffered from rheumatoid arthritis were excluded from participation. In addition, patients in whom the dysplasia may have been affected by a neurological illness or Legg-Calvé-Perthes disease were also excluded. There were 32 cases of bilateral and 31 cases of unilateral $\mathrm{AD}$. A total of 10 patients underwent a bilateral THA. Three-dimensional computed tomography (3DCT) was used to clarify whether a deformity existed and, if the result was positive, to identify the degree of acetabular or femoral deformity $(11,12)$. A total of 30 acetabula or femurs were not able to be located in the normal anatomical sites, due to a significant acetabular or femoral deformity, out of 73 dysplastic hips. The study was conducted in accordance with the Declaration of Helsinki and with approval from the Ethics Committee of Shanghai Sixth People's Hospital. Written informed consent was obtained from all participants.

Radiographic evaluation. The radiographic evidence of $\mathrm{AD}$ included a central-edge angle of Wiberg (CE angle) $<20^{\circ}$ on the anteroposterior radiographs (13), and a Sharp angle $>45^{\circ}$ for the Crowe type I subluxation (14). In Crowe type I DDH, the vertical subluxation of the hip (measured from the inferior margin of the tear drop to the head-neck junction) is $<50 \%$ of the diameter of the femoral head (or $<10 \%$ of the height of the pelvis) (7). CT scans were acquired at a thickness of $1.2 \mathrm{~mm}$, and a table speed of $3.0 \mathrm{~mm} / \mathrm{s}$, using a helical scanner (GE Lightspeed 16 Slice CT scanner, GE Healthcare, Waukesha, WI, USA). The helical scanning was conducted at $140 \mathrm{kVp}$ and $300 \mathrm{mAs}$, and the field of view was $500 \mathrm{~mm}$. Classifying the abnormalities using 3DCT involved basic scanning, ranging from $5 \mathrm{~cm}$ above the acetabular roof to the femoral condyles. The CT data were transferred digitally to Digital Imaging and Communications in Medicine (DICOM, version 3.0; National Electrical Manufacturers' Association, Rosslyn, VA, USA), where the images were formatted (512x512 pixels), prior to the retrieval of the images using a compact disc or a

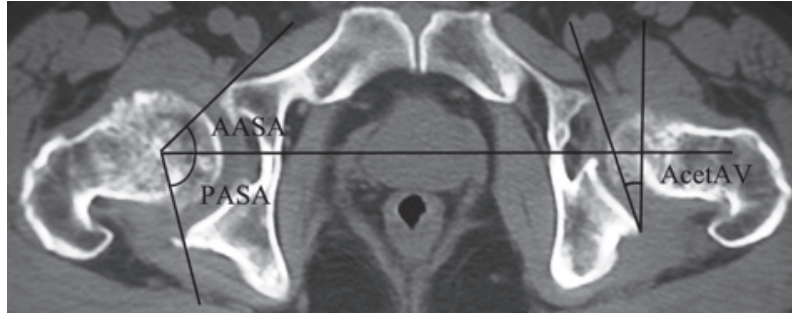

Figure 1. Reformatted axial image on which the acetabular anteversion angle (AcetAV), the anterior acetabular section angle (AASA) and the posterior acetabular section angle (PASA) passing through the center of the femoral heads were measured.

digital versatile disc. These retrieved data were transferred to a personal laptop computer (IBM Lenovo Thinkpad X220i, Lenovo, Inc., Beijing, China), and the 3D bone images of the acetabulum and femur were reconstructed and analyzed using Intage Realia software (KGT, Inc., Tokyo, Japan). The original data were reconstructed in $1 \mathrm{~mm}$ intervals on coronal and sagittal images of the hip joint (12). Two experienced hip surgeons, who were responsible for performing $>200$ cases each year, subsequently measured the following parameters, twice (8): i) Anterior acetabular section angle (AASA), i.e. the angle between the centerline extending between the bilateral femoral heads, and the line from the center of the head to the anterior margin of the acetabulum $\left(59-83^{\circ}\right.$ and $53-92^{\circ}$ in normal males and females, respectively (12); Fig. 1); ii) posterior acetabular section angle (PASA), i.e. the angle between the centerline extending between the bilateral femoral heads, and the line from the center of the head to the posterior margin of the acetabulum [84-116 ${ }^{\circ}$ and $87-120^{\circ}$ in normal males and females, respectively (12); Fig. 1)]; iii) acetabular anteversion angle (AcetAV), i.e. the angle between the line extending between the anterior and posterior margins of the acetabulum, and the line perpendicular to the center line connecting the bilateral femoral heads (12) (Fig. 1); iii) femur anteversion angle (FAVA) (15) (Fig. 2); iv) canal rotation angle (CRA), i.e. the angle between the major axis of the ellipses of best fit to the endosteal surface of the femoral canal, and a tangent to the posterior aspect of the femoral condyles $(4,11)$ (Fig. 3); v) medio-lateral and vi) antero-posterior canal width at the level of the canal isthmus (the maximum value of the medio-lateral or antero-posterior extracortical diameter of the diaphysis was also recorded); and vii) canal diameter at the isthmus (the point of the medullary canal with the smallest cross-sectional area). The mean of the normal population was used as the control $(4,11)$. Forty-six healthy controls with normal hip anatomy were also assessed, including 11 males and 35 females, with a mean age of $56.7 \pm 11.7$ years (range, 25-80 years).

Classification. The acetabular abnormalities were classified into A1-type anterior, A2-type posterior and A3-type lateral (including mild and global) deficiencies (Table I) (12). The femoral classification was as follows: F1-type, FAVA $<30^{\circ}$; F2-type, $30^{\circ} \leq$ FAVA $\leq 40^{\circ}$, with mild abnormalities of the femoral canal rotation and the diameter at the isthmus; F3-type, FAVA $>40^{\circ}$, with significant abnormalities of the femoral canal rotation and the diameter at the isthmus (Tables II and III). 

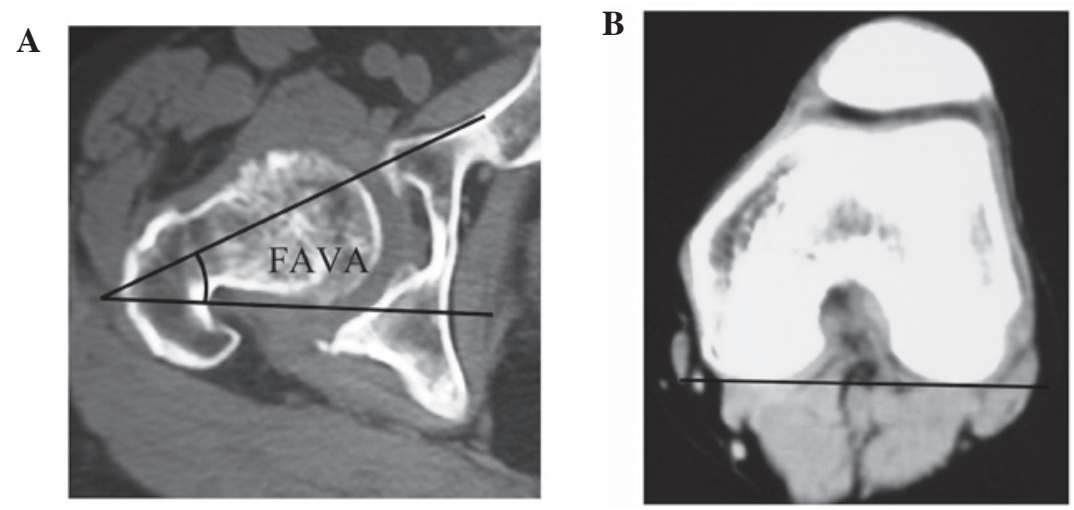

Figure 2. Femoral anteversion angle (FAVA) was defined as the angle between the femoral neck axis (A) and the transepicondylar axis (B).
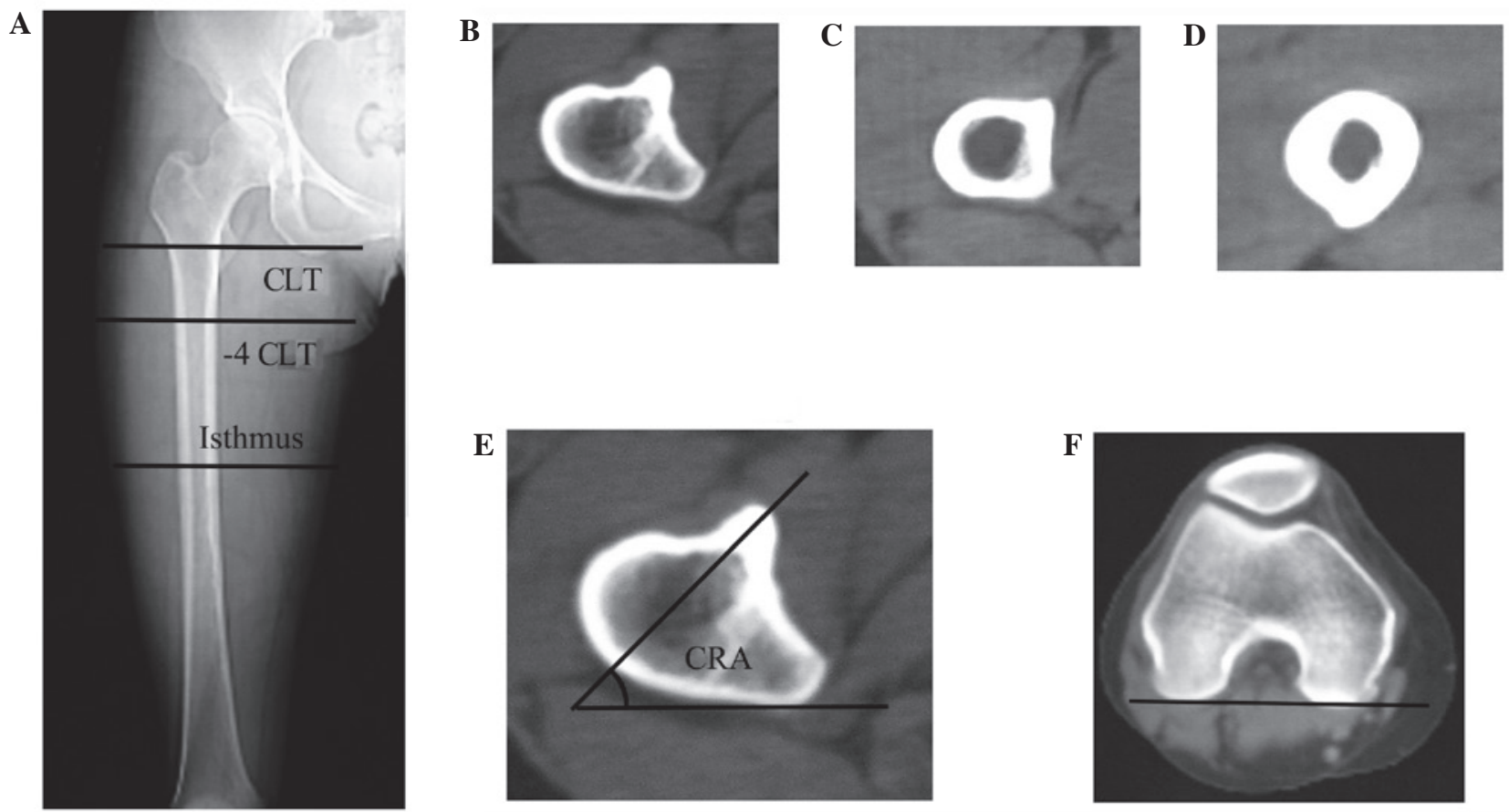

Figure 3. (A) Canal rotation angle (CRA) at three different cross sections of the femoral canal: (B) center of the lesser trochanter (CLT); (C) CLT-4 cm, $4 \mathrm{~cm}$ below the CLT; and (D) isthmus. (E) and (F) CRA: the angle between the major axis of the ellipses of best fit to the endosteal surface of the femoral canal and a tangent to the posterior aspect of the femoral condyles.

There were 21 A1-type cases (26 hips), nine A2-type cases (13 hips) and 33 A3-type cases (34 hips). In addition, there were 33 F1-type cases (35 hips), 26 F2-type cases (32 hips) and four F3-type cases (six hips).

Statistical analysis. The database was established via statistical analysis using SPSS 19.0 (SPSS, Inc., Chicago, IL, USA). For variables that were normally distributed, differences between the types were evaluated using analysis of variance (ANOVA), followed by the unpaired t-test for multiple pair-wise comparisons of all significant variables. Categorical data were compared using the $\chi^{2}$ test. To assess the intraobserver reliability of the different parameters of the femur or the acetabulum, the preoperative radiographs for each patient were templated by an investigator, who subsequently repeated the templating two weeks later. In addition, the templating procedure was repeated by a second investigator, independently.
The intra- and interobserver effects were calculated using an intraclass correlation coefficient (ICC) (8). Pearson's correlation coefficient was used to assess the correlations between various measurements. $\mathrm{P}<0.05$ was considered to indicate a statistically significant difference (Tables II and III).

\section{Results}

When the acetabular and femoral abnormalities were divided into subgroups, using 3DCT, it was observed that there was a crossover between each of the femoral subtypes (F1, F2 and F3) and the acetabular subtypes (A1, A2, or A3), with the exception that the F3-type deficiency did not appear in conjunction with the A2-type deficiency. Significant differences were demonstrated in the AcetAV $(\mathrm{P}<0.05)$, AASA $(\mathrm{P}<0.05)$ and PASA $(\mathrm{P}<0.05)$ between the $\mathrm{A} 1, \mathrm{~A} 2$ and $\mathrm{A} 3$-type deficiencies (A1 versus $\mathrm{A} 2, \mathrm{~A} 1$ versus $\mathrm{A} 3$ and $\mathrm{A} 2$ versus $\mathrm{A} 3$ ); 
Table I. Classification of acetabular dysplasia.

\begin{tabular}{lcccc} 
& \multirow{2}{*}{$\begin{array}{c}\text { A1-type anterior } \\
\text { deficiency }\end{array}$} & $\begin{array}{c}\text { A2-type posterior } \\
\text { deficiency }\end{array}$ & Mild deficiency & Global deficiency \\
\cline { 4 - 5 } & $<50^{\circ}$ & $\geq 50^{\circ}$ & $\geq 50^{\circ}$ & $<50^{\circ}$ \\
AASA & $\geq 90^{\circ}$ & $<90^{\circ}$ & $\geq 90^{\circ}$ & $<90^{\circ}$ \\
\hline
\end{tabular}

AASA, anterior acetabular section angle; PASA, posterior acetabular section angle.

Table II. Comparison of computed tomography measurements among the different types of acetabular deficiency.

\begin{tabular}{lcccccc}
\hline Group & $\mathrm{n}$ & $\mathrm{CE}$ angle $\left(^{\circ}\right)$ & Sharp angle $\left(^{\circ}\right)$ & AcetAV $\left(^{\circ}\right)$ & AASA $\left(^{\circ}\right)$ & $\operatorname{PASA}\left({ }^{\circ}\right)$ \\
\hline A1-type & 26 & $12.7 \pm 7.1^{\mathrm{a}}$ & $50.2 \pm 3.1^{\mathrm{a}}$ & $22.5 \pm 1.8^{\mathrm{a}}$ & $48.3 \pm 2.4^{\mathrm{a}}$ & $93.3 \pm 6.0$ \\
A2-type & 13 & $13.5 \pm 4.2^{\mathrm{a}}$ & $49.9 \pm 4.3^{\mathrm{a}}$ & $14.0 \pm 3.4^{\mathrm{a}}$ & $60.2 \pm 3.1^{\mathrm{a}}$ & $78.2 \pm 4.0^{\mathrm{a}}$ \\
A3-type & 34 & $11.9 \pm 5.7^{\mathrm{a}}$ & $52.1 \pm 5.0^{\mathrm{a}}$ & $19.6 \pm 4.6$ & $54.6 \pm 8.5^{\mathrm{a}}$ & $88.4 \pm 10.1^{\mathrm{a}}$ \\
Control & 46 & $31.0 \pm 4.3$ & $35.9 \pm 2.9$ & $19.8 \pm 3.7$ & $75.9 \pm 8.6$ & $95.3 \pm 6.0$ \\
\hline
\end{tabular}

Data are presented as the mean \pm standard deviation. ${ }^{a} \mathrm{P}<0.05$ compared with control. $\mathrm{CE}$ angle, central-edge angle of Wiberg; AcetAV, acetabular anteversion angle; AASA, anterior acetabular section angle; PASA, posterior acetabular section angle.

Table III. Anatomical parameters of control and dysplastic femurs, based on the different types of acetabular dysplasia.

\begin{tabular}{|c|c|c|c|c|}
\hline Parameters & $\begin{array}{c}\text { Control } \\
(n=46)\end{array}$ & $\begin{array}{l}\text { F1-type } \\
(n=35)\end{array}$ & $\begin{array}{c}\text { F2-type } \\
(n=32)\end{array}$ & $\begin{array}{c}\text { F3-type } \\
\quad(n=6)\end{array}$ \\
\hline Medio-lateral canal width at isthmus (mm) & $12.4 \pm 1.4$ & $12.3 \pm 1.5$ & $11.7 \pm 1.3^{\mathrm{a}}$ & $11.1 \pm 0.5^{\mathrm{a}}$ \\
\hline Antero-posterior canal width at isthmus (mm) & $13.6 \pm 1.4$ & $13.4 \pm 1.6$ & $12.8 \pm 1.3^{\mathrm{a}}$ & $12.2 \pm 0.7^{\mathrm{a}}$ \\
\hline Canal diameter at isthmus (mm) & $10.3 \pm 1.4$ & $10.4 \pm 1.7$ & $9.7 \pm 1.2^{\mathrm{a}}$ & $8.9 \pm 0.4^{\mathrm{a}}$ \\
\hline \multicolumn{5}{|l|}{ Canal rotation angle $\left({ }^{\circ}\right)$} \\
\hline At CLT & $45.2 \pm 3.7$ & $46.4 \pm 2.7$ & $48.6 \pm 2.0^{\mathrm{a}}$ & $52.1 \pm 2.1^{\mathrm{b}}$ \\
\hline CLT-4 cm & $49.8 \pm 3.4$ & $50.7 \pm 2.7$ & $54.0 \pm 3.2^{\mathrm{a}}$ & $58.6 \pm 1.1^{\mathrm{b}}$ \\
\hline At isthmus & $85.2 \pm 3.6$ & $84.1 \pm 2.5$ & $82.4 \pm 1.6^{\mathrm{a}}$ & $79.8 \pm 1.8^{b}$ \\
\hline $\operatorname{FAVA}\left({ }^{\circ}\right)$ & $18.6 \pm 5.0$ & $25.8 \pm 1.5^{\mathrm{a}}$ & $32.2 \pm 2.5^{\mathrm{a}}$ & $45.0 \pm 3.7^{\mathrm{b}}$ \\
\hline
\end{tabular}

Data are presented as the mean \pm standard deviation. ${ }^{\mathrm{a}} \mathrm{P}<0.05$ compared with control; ${ }^{\mathrm{b}} \mathrm{P}<0.05$ compared with $\mathrm{F} 2$-type deficiency. F1-type, femur anteversion angle (FAVA) $<30^{\circ} ; \mathrm{F} 2$-type, FAVA $\leq 40^{\circ}$; F3-type, FAVA $>40^{\circ}$. CLT, center of the lesser trochanter.

however, no significant differences were observed in the $\mathrm{CE}$ angle $(\mathrm{P}>0.05)$ or the Sharp angle $(\mathrm{P}>0.05)$. The AASA values of the A1, A2 and A3-type deficiencies were significantly different from that of the control group $(\mathrm{P}<0.05)$, whereas only the PASA values of the A2 and A3-type deficiencies were significantly different in comparison with the PASA value of the control group $(\mathrm{P}<0.05$; Table II). There was a significant Pearson's correlation between the AASA and the AcetAV $(r=-0.353, P=0.002)$, and between the PASA and the AcetAV $(\mathrm{r}=0.5, \mathrm{P}=0.001)$, indicating that hips with a greater AASA also had a lower AcetAV, and that those with a greater PASA also had a higher AcetAV. No significant differences were observed in the AcetAV between the A3-type deficiency and the control $(\mathrm{t}=0.102, \mathrm{P}=0.92)$. The intra- and interobserver reli- ability values of the acetabular classification, obtained using ICC, were 0.843 and 0.862 , respectively, which indicated good reproducibility in the acetabular measurements.

Table III displays the canal width at the level of the isthmus in the antero-posterior and medio-lateral directions, and the canal diameter at the isthmus; significant differences were observed between the control and the F2 and F3-type deficiencies $(\mathrm{P}<0.05)$, but not between the control and the F1-type deficiency $(\mathrm{P}>0.05)$. There was no significant difference in the canal diameter at the isthmus between the F2 and F3-type deficiencies $(\mathrm{P}=0.336)$, although the mean diameter of the canal of the F3-type femurs was smaller than that of the F2-type femurs (8.9 mm versus $9.7 \mathrm{~mm}$ ). The CRAs at the three levels were significantly different between the F2 and F3-type deficiencies 
$(\mathrm{P}<0.05)$. There were no significant differences in the CRAs between the F1-type deficiency and the control cases; however, significant differences were observed in a comparison between the F2 and F3-type deficiencies and the control $(\mathrm{P}<0.05)$. From the center of the lesser trochanter (CLT) to the medullary cavity of the isthmus, a gradual increase was observed in the CRA. However, it was observed that there was a significantly higher mean increase in the CRA from the CLT to the isthmus in the control cases $\left(40^{\circ}\right)$, in comparison with that of the F2 $\left(34^{\circ}\right)$ and F3 $\left(28^{\circ}\right)$-type deficiencies. The variation in femoral anteversion in the F3-type deficiency was of a greater significance than that in the F1 and F2-type hips $(\mathrm{P}<0.05)$. It was observed that femurs with a greater FAVA also appeared to have narrower canals $(\mathrm{r}=-0.315, \mathrm{P}=0.007)$, and a smaller CRA at the isthmus $(\mathrm{r}=-0.696, \mathrm{P}=0.007)$.

There was no significant correlation between the FAVA and the AcetAV in the dysplastic hips overall $(r=0.001, P=0.996)$. However, when the hips were divided into subgroups, a significant positive correlation was observed between the FAVA and the AcetAV in the anterior deficiency subgroups $(r=0.394$, $\mathrm{P}=0.046$ ). By contrast, there was no significant correlation between the FAVA and the AcetAV in the posterior and global deficiency subgroups $(\mathrm{r}=-0.006, \mathrm{P}=0.973$; and $\mathrm{r}=0.038$, $\mathrm{P}=0.829$, respectively). The intra- and interobserver reliability values of the femoral classification, obtained using ICC, were 0.813 and 0.822 , respectively, which indicated that there was an acceptable reliability in the femoral measurements. There were no significant differences in the average age $(\mathrm{t}=0.585$, $\mathrm{P}=0.561)$ or gender $\left(\chi^{2}=0.040, \mathrm{P}=0.836\right)$ of the 63 patients with AD compared with the 46 healthy controls. In the control hips, no significant correlations were observed between the FAVA and the AcetAV, Sharp angle or CE angle $(r=-0.115$, $\mathrm{P}=0.448 ; \mathrm{r}=0.041, \mathrm{P}=0.785 ;$ and $\mathrm{r}=0.026, \mathrm{P}=0.078$, respectively). However, there was a significant positive correlation between the FAVA and the Sharp angle ( $r=0.456, \mathrm{P}=0.00)$, and a significant negative correlation between the FAVA and the $\mathrm{CE}$ angle $(\mathrm{r}=-0.473, \mathrm{P}=0.00)$ in the dysplastic hips.

\section{Discussion}

In this study of 73 dysplastic hips and 46 normal hips, the morphological differences between dysplastic and normal hips were observed, and significant correlations between the AcetAV and the acetabular anterior or posterior deficiency subgroups were identified. In addition, it was demonstrated that there was a significant correlation betwen the femoral anteversion and the AcetAV in the anterior deficiency subgroup. It was revealed by Akiyama et al (5) that changes in the AASA, PASA and AcetAV may be detected by 3DCT, and that 3DCT clearly exhibits the location and extent of the dysplasia. In a study by Ito et al (12), 22 of 84 AD hips (26\%) were classified as having an anterior deficiency; 17 (20\%), a posterior deficiency; and 45 (54\%), a lateral deficiency. Hips with poor anterior acetabular support were defined as those with an AASA $<50^{\circ}$, while hips with poor posterior support were defined as those with a PASA $<90^{\circ}$ (12). In a previous study, the AASA, PASA, and AcetAV measurements were demonstrated to be effective for the precise evaluation of various acetabular deficiencies (16). Anda et al (17) revealed that the AcetAV in the anterior deficiency subgroup was significantly larger than in the other groups. By contrast, the AcetAV in the posterior deficiency subgroup has been observed to be smaller than that in the normal and global deficiency subgroups (5). The results of these studies supported the observations in the present study. In addition, the results of the present study demonstrated a trend towards increased or decreased acetabular anteversion in shallow hips with poor anterior or posterior support.

The previously mentioned results indicated the existence of a potential developmental interaction between the femur and acetabulum. When the dysplastic hips were divided according to the location of the acetabular bone defect, significant differences in acetabular version were observed among the subgroups. It was demonstrated that hips with a larger FAVA appeared to additionally have an increased AcetAV, indicating a biomechanical cycle resulting in the pathology of dysplastic hips with anterior acetabular deficiency (5). By contrast, no correlation in version was observed in hips with a posterior or global deficiency. However, it was demonstrated that there was a significant correlation between the FAVA and the Sharp/CE angles in the dysplastic acetabula.

Although the previous studies indicated that each type of dysplasia was correlated with the degree of the dysplasia, rather than the specific type of severity, they did not offer a systematic and detailed guide for THA. The large individual acetabular morphological variability across all levels of dysplasia observed in this study demonstrated that it is not possible to select an acetabular prosthesis for dysplastic hips on the basis of the severity of the subluxation alone. The results of the study suggested that there is a requirement for the surgeon to choose the type of socket implantation according to the type and extent of the acetabular defect, and to adapt to the individual FAVA. Thus, it is necessary for each patient be considered individually, in order that the angle of the acetabular cup may be customized to suit (9). For the A1-type deficiency, a reduction in the AcetAV or a neutral position is required when the cup is implanted. In cases with an excessive FAVA, a decrease in the FAVA is required for the inclusion and congruity of the hip joint (5). For the A2-type deficiency, an appropriate increase in the AcetAV is required to resolve the initial instability, in order to prevent the aggravation of the posterior acetabular insufficiency. In the present study, no significant differences were found in the AcetAV between the A3-type deficiency (mild or global) and the control group. The acetabular defects predominantly occurred on the upper and lateral margins of the acetabulum, although anterior and posterior deficiencies were also observed with the global deficiency. Due to the absence of structural bone defects in the acetabula, and since the acetabular cup covers $>70 \%$ of the bone bed, there is a requirement for the acetabular cup to be placed at the center of the acetabulum, and for normal anteversion to be maintained (2). Since mild or global deficiencies of the acetabulum require similar methods of prosthetic implantation, hips with these types of deficiency may be classified as having a lateral deficiency (12). The treatment of femoral abnormalities or variations with A1, A2 or A3-type deficiencies are described in greater detail later in this study.

The present study revealed the morphological characteristics of dysplastic femurs, and investigated the effects of the disorder on the geometry of the intramedullary canal. These results were then compared with a control group. It was demon- 
strated that in cases with excessive anteversion, the dysplastic femurs were smaller than the control femurs, with narrower, straighter and less-tapered canals. Sugano et al and Noble et al $(4,11)$ observed that the 3D anatomy of a femur with the mildest degree of subluxation (Crowe type I) exhibited a significantly different FAVA and medullary cavity rotation, and that several patients had a FAVA $>60^{\circ}$. In addition, it was demonstrated that the diameter of the femoral medullary cavity was reduced in the Crowe type I femurs. The minimum diameter of the canal in the Crowe type I femurs was $1 \mathrm{~mm}$ less than in the control femurs $(4,11)$. Argenson et al $(9)$ revealed that the mean diameter of the medullary cavity was $>1.6 \mathrm{~mm}$ narrower in the antero-posterior and $>1.9 \mathrm{~mm}$ narrower in the medio-lateral position in the Crowe type I than in the control femurs $(9,11)$. The canal flare and metaphyseal canal flare indices were used to assess variations in the width of the femoral medullary cavity on anteroposterior radiographs (18). X-rays are only able to assess the femoral marrow cavity in two dimensions; however, with the exception of the differences in canal width, it is important that the morphological characteristics of the femoral medullary cavity at different levels are identifiable with 3DCT. Therefore, the measurements were performed in three dimensions, i.e. in the axial, coronal and sagittal planes (19). It was observed that the normal rotation angle of the medullary canal gradually increased from the CLT to the isthmus. However, in the dysplastic femurs, the increased anteversion of the proximal femur resulted in a reduction in the rotation in the medullary canal, predominantly in the region from the CLT to the canal at the isthmus $(4,11)$. The variation between the F2 and F3-type deficiencies supported this observation. With regard to surgery, this variation is critical, since it is necessary to be aware of variations in the width of the medullary cavity when the femoral canal is reamed, in order to avoid femoral fractures. When the femoral stem is implanted, there is a requirement for the morphological differences that occur at different levels of the medullary cavity to be considered, in order to ensure that the prosthesis closely matches the medullary cavity of the femur. Therefore, when the FAVA is exaggerated, the rotational orientation has a marked effect on the size and shape of the canal (11), and the concomitant twist of the femoral canal increases the difficulty of the joint replacement.

The results of the present study demonstrated that the position of the femoral anterior arch in the femurs with AD was not significantly different from that observed in the control group. This indicated that the primary anatomical feature affecting the successful placement of the stem is increased femur anteversion, leading to secondary rotational anomalies and a narrowing of the canal at the isthmus. Therefore, these features were the basis of our classification $(4,9)$. With regard to the F1-type deficiency (FAVA $<30^{\circ}$ ), femoral stem implantation with a normal FAVA is possible. For the F2-type deficiency $\left(30^{\circ} \leq \mathrm{FAVA}<40^{\circ}\right.$, with mild abnormalities of the femoral canal rotation and the isthmus diameter) it may be appropriate to adjust the FAVA from $15^{\circ}$ to $25^{\circ}$, due to the anteversion of the acetabular cup. However, following femoral neck osteotomy, the cross-section of the long axis of the femur is not usually consistent with that required by the femoral stem. If a proximal fixed prosthesis is chosen, stability is poor; therefore, in the majority of cases a prosthesis with a straight and thin distal stem is required to accommodate this diaphyseal femoral anatomy (20). With regard to F3-type deficiencies, with significant abnormalities of the femoral canal rotation and a reduced isthmus diameter, it has been demonstrated that modular or customized components are necessary, in order to accommodate the shape of these dysplastic canals $(21,22)$. Furthermore, the present study indicated that the templating technique exhibited the desired reliability, with all the ICC values exceeding 0.8 (8).

A retrospective database and image review was used to summarize the diversity of mild dysplasia; this reinforced the observations of a number of previous studies, concerning the exaggerated anteversion in mildly dysplastic femurs. At present, the majority of doctors do not consider the disorder of mild dysplasia to be a great disability, and, furthermore, preoperative $3 \mathrm{DCT}$ scans are not routinely requested for Crowe type I hips, due to the additional medical expense. However, the present study revealed the anatomical variations of the acetabulum and proximal femoral medullary cavity to be irregular and interrelated ( $41.1 \%$ of cases), and preoperative X-rays and 2DCT scans of the hip joint are not able to identify any correlation between these variations. It is therefore important that the results of 3D scans are assessed preoperatively, and that any interrelation between the femoral and acetabular morphologies is identified by the surgeons. The aim of this investigation was to emphasize the morphological variations in mild dysplasia, particularly in the femoral medullary cavity and the acetabulum, as a primary step to determining the potential requirements for surgical procedures. The results of this study are likely to provide a greater insight into the morphological characteristics of dysplastic hips, and the challenges confronting joint replacement surgeons.

In addition to suggesting a novel anatomic classification, this study provided a detailed characterization of the anatomical variations to be considered in hip arthroplasty implants for patients with AD. The purpose of this 3D morphometric analysis was to serve as an anatomical reference for acetabular and femoral implants. The novel classification employed in this study used 3DCT measurements to clarify the location and extent of acetabular deficiency, the diameter of the medullary cavity at the isthmus and the degree of rotational deformity. This is likely to facilitate the improved management of malformations of the acetabulum and femur, and to ensure the selection of a suitable prosthesis. Since the initial assessment of the patients with AD has been adopted, the individualized prosthesis implantation and surrounding bone matching have achieved the desired results, thereby increasing the long-term survival rates of the prostheses.

\section{Acknowledgements}

This study was supported by the Interdisciplinary (Engineering-Medical) Research Fund of Shanghai Jiao Tong University (grant no. YG2011MS30), Shanghai Municipal Health Bureau Science Fund for Young Scholars (grant no. 2010QJ036A), the Opening Project of the State Key Laboratory of High Performance Ceramics and Superfine Microstructure (grant no. SKL201206SIC) and the National Natural Science Foundation of China (grant no. 81171688). 


\section{References}

1. Hartofilakidis G, Yiannakopoulos CK and Babis GC: The morphologic variations of low and high hip dislocation. Clin Orthop Relat Res 466: 820-824, 2008.

2. Xu WD, Li J, Zhou ZH, Wu YS and Li M: Results of hip resurfacing for developmental dysplasia of the hip of Crowe type I and II. Chin Med J (Engl) 121: 1379-1383, 2008.

3. Jasty M, Anderson MJ and Harris WH: Total hip replacement for developmental dysplasia of the hip. Clin Orthop Relat Res 311: 40-45, 1995.

4. Sugano N, Noble PC, Kamaric E, Salama JK, Ochi T and Tullos HS: The morphology of the femur in developmental dysplasia of the hip. J Bone Joint Surg Br 80: 711-719, 1998.

5. Akiyama M, Nakashima Y, Fujii M, et al: Femoral anteversion is correlated with acetabular version and coverage in Asian women with anterior and global deficient subgroups of hip dysplasia: a CT study. Skeletal Radiol 41: 1411-1418, 2012.

6. Spangehl MJ, Berry DJ, Trousdale RT and Cabanela ME: Uncemented acetabular components with bulk femoral head autograft for acetabular reconstruction in developmental dysplasia of the hip: results at five to twelve years. J Bone Joint Surg Am 83-A: 1484-1489, 2001.

7. Jawad MU and Scully SP: In brief: Crowe's classification: arthroplasty in developmental dysplasia of the hip. Clin Orthop Relat Res 469: 306-308, 2011.

8. Yiannakopoulos CK, Chougle A, Eskelinen A, Hodgkinson JP and Hartofilakidis G: Inter- and intra-observer variability of the Crowe and Hartofilakidis classification systems for congenital hip disease in adults. J Bone Joint Surg Br 90: 579-583, 2008.

9. Argenson JN, Flecher X, Parratte S and Aubaniac JM: Anatomy of the dysplastic hip and consequences for total hip arthroplasty. Clin Orthop Relat Res 465: 40-45, 2007.

10. Shen B, Yang J, Wang L, Zhou ZK, Kang PD and Pei FX: Midterm results of hybrid total hip arthroplasty for treatment of osteoarthritis secondary to developmental dysplasia of the hip-Chinese experience. J Arthroplasty 24: 1157-1163, 2009.

11. Noble PC, Kamaric E, Sugano N, Matsubara M, Harada Y, Ohzono $\mathrm{K}$ and Paravic V: Three-dimensional shape of the dysplastic femur: implications for THR. Clin Orthop Relat Res 417: 27-40, 2003.
12. Ito H, Matsuno T, Hirayama T, Tanino H, Yamanaka $Y$ and Minami A: Three-dimensional computed tomography analysis of non-osteoarthritic adult acetabular dysplasia. Skeletal Radiol 38: 131-139, 2009.

13. Paliobeis CP and Villar RN: The prevalence of dysplasia in femoroacetabular impingement. Hip Int 21: 141-145, 2011.

14. Omeroglu H, Bicimoglu A, Agus H and Tumer Y: Acetabular development in developmental dysplasia of the hip. A radiographic study in anatomically reduced and uncomplicated hips. Bull NYU Hosp Jt Dis 65: 276-279, 2007.

15. Sugano N, Noble PC and Kamaric E: A comparison of alternative methods of measuring femoral anteversion. J Comput Assist Tomogr 22: 610-614, 1998.

16. Tallroth K and Lepistö J: Computed tomography measurement of acetabular dimensions: normal values for correction of dysplasia. Acta Orthop 77: 598-602, 2006.

17. Anda S, Terjesen T, Kvistad KA and Svenningsen S: Acetabular angles and femoral anteversion in dysplastic hips in adults: CT investigation. J Comput Assist Tomogr 15: 115-120, 1991.

18. Casper DS, Kim GK, Parvizi J and Freeman TA: Morphology of the proximal femur differs widely with age and sex: relevance to design and selection of femoral prostheses. J Orthop Res 30: 1162-1166, 2012.

19. Memon AR, Butler J, Guerin S, Galbraith J, Flanagan O and Harty J: Proximal femoral anatomy in total hip arthroplasty. A tri-planar computed tomographic assessment. Acta Orthop Belg 77: 488-493, 2011.

20. Schmidutz F, Beirer M, Weber P, Mazoochian F, Fottner A and Jansson V: Biomechanical reconstruction of the hip: comparison between modular short-stem hip arthroplasty and conventional total hip arthroplasty. Int Orthop 36: 1341-1347, 2012.

21. Ito $H$, Tanino $H$, Yamanaka $Y$, Nakamura $T$ and Matsuno $T$ : Hybrid total hip arthroplasty using specifically-designed stems for patients with developmental dysplasia of the hip. A minimum five-year follow-up study. Int Orthop 35: 1289-1294, 2011.

22. Sakai T, Sugano N, Ohzono K, Nishii T, Haraguchi K and Yoshikawa H: Femoral anteversion, femoral offset, and abductor lever arm after total hip arthroplasty using a modular femoral neck system. J Orthop Sci 7: 62-67, 2002. 